Tôhoku Math. Journ.

29 (1977), 275-285.

\title{
THE EQUIVALENCE OF TWO CONDITIONS ON WEIGHTED NORM INEQUALITIES FOR MARTINGALES
}

\author{
N. KAZAMAKI
}

(Received April 12, 1976)

0. Introduction. Let $(\Omega, F, P)$ be a complete probability space and let $\left(F_{t}\right)$ be a non-decreasing right continuous family of sub $\sigma$-fields of $F$ with $\bigvee_{t \geq 0} F_{t}=F$ such that $F_{0}$ contains all $P$-null sets. Now, fix a random variable $Z$ such that $Z>0$ a.s., and $E[Z]=1$, and define $Z_{t}=$ $E\left[Z \mid F_{t}\right]$. In this paper we assume that the martingale $Z_{t}$ is continuous and $Z_{0}=1$. Then, as is well known, there exists a unique continuous local martingale $M$ with $M_{0}=0$ such that $Z_{t}=\exp \left(M_{t}-(1 / 2)\langle M\rangle_{t}\right)$. Here $\langle M\rangle$ denotes the unique continuous increasing process such that $M^{2}-\langle M\rangle$ is a local martingale. This representation is of fundamental importance in our study. Furthermore we assume that the local martingale $M$ is $L^{2}$-bounded. We proved in [1] that $M$ is a $B M O$-martingale if and only if $Z$ satisfies the condition:

$$
\sup _{t} \text { ess. sup } E\left[\left(\frac{Z_{t}}{Z}\right)^{1 /(p-1)} \mid F_{t}\right]^{p-1}<\infty
$$

for some $p>1$. We denote this supremum by $A_{p}(Z)$. On the other hand, $M$ is a $B M O$-martingale if and only if $Z$ satisfies the condition:

$$
\sup _{t} \operatorname{ess.\operatorname {sup}} E\left[\exp \left\{\lambda\left(\langle M\rangle_{\infty}-\langle M\rangle_{t}\right)\right\} \mid F_{t}\right]<\infty
$$

for some $\lambda>0$. For convenience, the supremum is denoted by $B_{\lambda}(Z)$. Our aim is to investigate the relation between the conditions $\left(A_{p}\right)$ and $\left(B_{\lambda}\right)$. In Section 2 we show that there exists an equivalence relation between these conditions. Namely, set $Z_{t}^{\prime}=\exp \left(-M_{t}-(1 / 2)\langle M\rangle_{t}\right)$ and $Z^{\prime}=Z_{\infty}^{\prime}$. Then in order that $Z$ satisfies $\left(B_{\lambda}\right)$ for all $\lambda>0$, it is necessary and sufficient that $Z$ and $Z^{\prime}$ satisfy $\left(\mathrm{A}_{\mathrm{p}}\right)$ for all $p>1$. In Section 3 we prove: if $M$ is a $B M O$-martingale, there exists a non-decreasing sequence $\left(T_{n}\right)$ of stopping times with $\lim _{n} T_{n}=\infty$ a.s., such that the weighted norm inequality

$$
\int \sup _{\Omega 0 \leqq t T_{n}}\left|X_{t}\right|^{p} Z d P \leqq C_{p, n} \int_{\Omega}\left|X_{T_{n}}\right|^{p} Z d P, \quad 1<p<\infty, \quad n=1,2, \cdots
$$

is valid for all uniformly integrable martingale $X$. Here $C_{p, n}$ is a con- 
stant independent of $X$. Finally, we apply these results to prove a new type martingale inequality. Namely, if $M$ is a $B M O$-martingale, then for every $p>1$ there exists a positive constant $c_{p}$ depending only on $p$ such that the inequality

$$
E\left[\sup _{t}\left|X_{t}-c_{p}\langle X, M\rangle_{t}\right|^{p}\right] \leqq C_{p} E\left[\left|X_{\infty}-c_{p}\langle X, M\rangle_{\infty}\right|^{p}\right]
$$

is valid for all $L^{2}$-bounded continuous martingale $X$. Particularly, if $Z$ satisfies $\left(\mathrm{B}_{\lambda}\right)$ for all $\lambda>0$, then $c_{p}$ may be taken to be 1 .

The reader is assumed to be familiar with the martingale theory as expounded in [2]. In what follows we denote by $C$ a positive constant and by $C_{p}$ a positive constant depending only on the indexed parameter $p$, both letters are not necessarily the same in each occurence.

1. The $\left(B_{\lambda}\right)$ condition. First, observe that $Z$ satisfies $\left(B_{\lambda}\right)$ if and only if for any stopping time $T$

$$
E\left[\exp \left\{\lambda\left(\langle M\rangle_{\infty}-\langle M\rangle_{T}\right)\right\} \mid F_{T}\right] \leqq C_{\lambda} \text { a.s. }
$$

For simplicity, we denote by $\left(\mathrm{B}_{\infty}\right)$ the condition such that the $\left(\mathrm{B}_{\lambda}\right)$ condition holds for some $\lambda>0$.

LEMma 1. If $Z$ satisfies $\left(\mathrm{B}_{\lambda}\right)$, then for any stopping time $T$

$$
E\left[\exp \left(\sqrt{\frac{\lambda}{2}}\left|M_{\infty}-M_{T}\right|\right) \mid F_{T}\right] \leqq 2 B_{\lambda}(Z)^{1 / 2} \quad \text { a.s. }
$$

Proof. Since exp $\left(\sqrt{2 \lambda} M_{t}-\lambda\langle M\rangle_{t}\right)$ is a local martingale, a trivial stopping argument and the Fatou lemma give

$$
E\left[\exp \left\{\sqrt{2 \lambda}\left(M_{\infty}-M_{T}\right)-\lambda\left(\langle M\rangle_{\infty}-\langle M\rangle_{T}\right)\right\} \mid F_{T}\right] \leqq 1 .
$$

An application of the Schwarz inequality shows that

$$
\begin{aligned}
E[\exp & \left.\left\{\sqrt{\frac{\lambda}{2}}\left(M_{\infty}-M_{T}\right)\right\} \mid F_{T}\right] \\
= & E\left[\exp \left\{\sqrt{\frac{\lambda}{2}}\left(M_{\infty}-M_{T}\right)-\frac{\lambda}{2}\left(\langle M\rangle_{\infty}-\langle M\rangle_{T}\right)\right\}\right. \\
& \left.\times \exp \left\{\frac{\lambda}{2}\left(\langle M\rangle_{\infty}-\langle M\rangle_{T}\right)\right\} \mid F_{T}\right] \\
\leqq & E\left[\exp \left\{\lambda\left(\langle M\rangle_{\infty}-\langle M\rangle_{T}\right)\right\} \mid F_{T}\right]^{1 / 2} \\
\leqq & B_{\lambda}(Z)^{1 / 2} \text { a.s. }
\end{aligned}
$$

The same argument works if $M$ is replaced by $-M$. Thus the lemma is established. 
A continuous local martingale $X$ is a $B M O$-martingale if

$$
\|X\|_{B M O}^{2}=\sup _{t} \operatorname{ess.sup} E\left[\langle X\rangle_{\infty}-\langle X\rangle_{t} \mid F_{t}\right]<\infty \text {. }
$$

LEMma 2. Let $X$ be a continuous martingale with $\|X\|_{\text {Bмo }}<1$. Then for any stopping time $T$

$$
E\left[\exp \left(\langle X\rangle_{\infty}-\langle X\rangle_{T}\right) \mid F_{T}\right] \leqq \frac{1}{1-\|X\|_{B M O}^{2}} \text { a.s. }
$$

Proof. To prove (2), it suffices to show that for every $A \in F_{T}$

$$
E\left[\exp \left(\langle X\rangle_{\infty}-\langle X\rangle_{T}\right) ; A\right] \leqq \frac{1}{1-\|X\|_{B M O}^{2}} P(A) \quad \text { a.s. }
$$

We may assume that $P(A)>0$. Let $d P^{\prime}=I_{A}(d P / P(A))$ and $F_{t}^{\prime}=F_{T+t}$. Then $X_{t}^{\prime}=X_{T+t}-X_{T}$ is a $P^{\prime}$-continuous martingale over $\left(F_{t}^{\prime}\right)$ such that $\left\langle X^{\prime}\right\rangle_{t}=\langle X\rangle_{T+t}-\langle X\rangle_{T}$. Clearly, we have

$$
E\left[\exp \left(\langle X\rangle_{\infty}-\langle X\rangle_{T}\right) ; A\right]=E^{\prime}\left[\exp \left(\left\langle X^{\prime}\right\rangle_{\infty}\right)\right] P(A)
$$

where $E^{\prime}[\cdot]$ denotes expectation over $\Omega$ with respect to $d P^{\prime}$. Now, let $S$ be any $F_{t}^{\prime}$-stopping time. Then for any $\Lambda \in F_{s}^{\prime}=F_{T+S}$ we get

$$
\begin{aligned}
\int_{.} E^{\prime}\left[\left\langle X^{\prime}\right\rangle_{\infty}-\left\langle X^{\prime}\right\rangle_{S} \mid F_{S}^{\prime}\right] d P^{\prime} & =\frac{1}{P(A)} \int_{A \cap A}\left(\langle X\rangle_{\infty}-\langle X\rangle_{T+S}\right) d P \\
& =\frac{1}{P(A)} \int_{A} E\left[\langle X\rangle_{\infty}-\langle X\rangle_{T+S} \mid F_{T+S}\right] I_{A} d P \\
& \leqq \int_{A}\|X\|_{B M o}^{2} d P^{\prime},
\end{aligned}
$$

from which $E^{\prime}\left[\left\langle X^{\prime}\right\rangle_{\infty}-\left\langle X^{\prime}\right\rangle_{S} \mid F_{S}^{\prime}\right] \leqq\|X\|_{B M O}^{2}$. By the energy inequalities,

$$
E^{\prime}\left[\left\langle X^{\prime}\right\rangle_{\infty}^{n}\right] \leqq n !\|X\|_{B M O}^{2 n}, \quad n=0,1,2, \cdots \text {. }
$$

As $\|X\|_{B M O}<1$, we get

$$
\begin{aligned}
E^{\prime}\left[\exp \left(\left\langle X^{\prime}\right\rangle_{\infty}\right)\right] & =\sum_{n=0}^{\infty} \frac{1}{n !} E^{\prime}\left[\left\langle X^{\prime}\right\rangle_{\infty}^{n}\right] \\
& \leqq \frac{1}{1-\|X\|_{B M O}^{2}},
\end{aligned}
$$

completing the proof.

From Lemma 2, $Z$ satisfies the $\left(\mathrm{B}_{1}\right)$ condition if $\|M\|_{B M O}<1$. More generally, if $M$ is a non-trivial $B M O$-martingale, then $Z$ satisfies the $\left(\mathrm{B}_{\lambda}\right)$ condition for $\lambda=1 /\left(2\|M\|_{B M O}^{2}\right)$. Conversely, if $Z$ satisfies $\left(\mathrm{B}_{\lambda}\right)$ for some $\lambda>0$, the Jensen inequality shows that 


$$
\|M\|_{B M O}^{2} \leqq \frac{1}{\lambda} \log \mathrm{B}_{\lambda}(Z),
$$

from which $M$ is a $B M O$-martingale. Consequently, $Z$ satisfies the $\left(\mathrm{B}_{\infty}\right)$ condition if and only if $M$ is a $B M O$-martingale. For example, $Z$ satisfies $\left(\mathrm{B}_{\lambda}\right)$ for all $\lambda>0$ if $\langle M\rangle$ is bounded.

2. The relation between $\left(A_{p}\right)$ and $\left(B_{\lambda}\right)$. If $Z$ satisfies $\left(A_{p}\right)$, then the Hölder inequality shows that it satisfies $\left(\mathrm{A}_{\mathrm{r}}\right)$ for every $r>p$. For convenience, we denote by $\left(A_{\infty}\right)$ the condition such that the $\left(A_{p}\right)$ condition holds for some $p>1$.

The main result of this section is the following.

THEOREM 1.

(i) $Z$ and $Z^{\prime}$ satisfy $\left(\mathrm{A}_{\mathrm{p}}\right)$ for all $p>1$ if and only if $Z$ satisfies $\left(\mathrm{B}_{\lambda}\right)$ for all $\lambda>0$.

(ii) The $\left(\mathrm{A}_{\infty}\right)$ condition is equivalent to the $\left(\mathrm{B}_{\infty}\right)$ condition.

This is an immediate consequence of the next lemma.

LEMma 3. Let $1<p<\infty$, and let $T$ be any stopping time.

(i) If $Z$ satisfies $\left(\mathrm{A}_{\mathrm{p}}\right)$, then

$$
\begin{aligned}
& E\left[\exp \left\{\frac{1}{2(\sqrt{p}+1)^{2}}\left(\langle M\rangle_{\infty}-\langle M\rangle_{T}\right)\right\} \mid F_{T}\right] \leqq A_{p}(Z)^{\sqrt{p}(\sqrt{p}+1)} \\
& E\left[\exp \left\{\frac{1}{2(\sqrt{p}+1)}\left|M_{\infty}-M_{T}\right|\right\} \mid F_{T}\right] \leqq 2 A_{p}(Z)^{\sqrt{p}(\sqrt{p}+1) / 2}
\end{aligned}
$$

(ii) If $Z$ and $Z^{\prime}$ satisfy $\left(\mathrm{A}_{\mathrm{p}}\right)$, then

$$
\begin{aligned}
& E\left[\exp \left\{\frac{1}{2(p-1)}\left(\langle M\rangle_{\infty}-\langle M\rangle_{T}\right)\right\} \mid F_{T}\right] \leqq\left\{A_{p}(Z) A_{p}\left(Z^{\prime}\right)\right\}^{1 / 2(p-1)} \\
& E\left[\exp \left\{\frac{1}{2 \sqrt{p-1}}\left|M_{\infty}-M_{T}\right|\right\} \mid F_{T}\right] \leqq 2\left\{A_{p}(Z) A_{p}\left(Z^{\prime}\right)\right\}^{1 / 4(p-1)}
\end{aligned}
$$

(iii) Conversely, if the inequality

$$
E\left[\exp \left\{\frac{1}{2(\sqrt{p}-1)^{2}}\left(\langle M\rangle_{\infty}-\langle M\rangle_{T}\right)\right\} \mid F_{T}\right] \leqq C_{p}
$$

holds, then $Z$ and $Z^{\prime}$ satisfy $\left(\mathrm{A}_{\mathrm{p}}\right)$.

Proof. According to Lemma 1, (4) follows at once from (3). Similarily, (5) implies (6). We begin with the proof of (3). Set $p_{0}=$ $\sqrt{p} /(\sqrt{p}-1)$. Then, as the exponent conjugate $q_{0}$ is $\sqrt{p}$, 


$$
\frac{1}{2 p_{0}(p-1)}-\frac{q_{0}}{2 p_{0}^{2}(p-1)^{2}}=\frac{1}{2(\sqrt{p}+1)^{2}} .
$$

We apply the Hölder inequality

$$
\begin{aligned}
E[\exp & \left.\left\{\frac{1}{2(\sqrt{p}+1)^{2}}\left(\langle M\rangle_{\infty}-\langle M\rangle_{T}\right)\right\} \mid F_{T}\right] \\
= & E\left[\exp \left\{-\frac{1}{p_{0}(p-1)}\left(M_{\infty}-M_{T}\right)+\frac{1}{2 p_{0}(p-1)}\left(\langle M\rangle_{\infty}-\langle M\rangle_{T}\right)\right\}\right. \\
& \left.\times \exp \left\{\frac{1}{p_{0}(p-1)}\left(M_{\infty}-M_{T}\right)-\frac{q_{0}}{2 p_{0}^{2}(p-1)^{2}}\left(\langle M\rangle_{\infty}-\langle M\rangle_{T}\right)\right\} \mid F_{T}\right] \\
\leqq & E\left[\exp \left\{-\frac{1}{p-1}\left(M_{\infty}-M_{T}\right)+\frac{1}{2(p-1)}\left(\langle M\rangle_{\infty}-\langle M\rangle_{T}\right)\right\} \mid F_{T}\right]^{1 / p_{0}} \\
& \times E\left[\operatorname { e x p } \left\{\frac{q_{0}}{p_{0}(p-1)}\left(M_{\infty}-M_{T}\right)\right.\right. \\
- & \left.\left.\frac{1}{2}\left(\frac{q_{0}}{p_{0}(p-1)}\right)^{2}\left(\langle M\rangle_{\infty}-\langle M\rangle_{T}\right)\right\} \mid F_{T}\right]^{1 / q_{0}} .
\end{aligned}
$$

As $Z_{T} / Z=\exp \left\{-\left(M_{\infty}-M_{T}\right)+(1 / 2)\left(\langle M\rangle_{\infty}-\langle M\rangle_{T}\right)\right\}$, the first term on the right side is equal to $E\left[\left(Z_{T} / Z\right)^{1 /(p-1)} \mid F_{T}\right]^{1 / p_{0}}$ which dominated by $A_{p}(Z)^{\sqrt{p}(\sqrt{p}+1)}$. The second term is also dominated by 1 . Consequently, (3) holds.

Now we are going to show (5). By the Schwarz inequality, we have

$$
\begin{aligned}
& E\left[\exp \left\{\frac{1}{2(p-1)}\left(\langle M\rangle_{\infty}-\langle M\rangle_{T}\right)\right\} \mid F_{T}\right] \\
& \leqq E\left[\exp \left\{-\frac{1}{p-1}\left(M_{\infty}-M_{T}\right)+\frac{1}{2(p-1)}\left(\langle M\rangle_{\infty}-\langle M\rangle_{T}\right)\right\} \mid F_{T}\right]^{1 / 2} \\
& \times E\left[\exp \left\{\frac{1}{p-1}\left(M_{\infty}-M_{T}\right)+\frac{1}{2(p-1)}\left(\langle M\rangle_{\infty}-\langle M\rangle_{T}\right)\right\} \mid F_{T}\right]^{1 / 2} .
\end{aligned}
$$

The first term on the right side is $E\left[\left(Z_{T} / Z\right)^{1 /(p-1)} \mid F_{T}\right]^{1 / 2}$, and the second term is $E\left[\left(Z_{T}^{\prime} / Z^{\prime}\right)^{1 /(p-1)} \mid F_{T}\right]^{1 / 2}$. Then (5) follows at once.

Finally, we prove (iii). Set $p_{0}=\sqrt{p}+1$. As $q_{0}=(\sqrt{p}+1) / \sqrt{p}$ and

$$
\frac{1}{p-1}=\frac{1}{q_{0}(\sqrt{p}-1)^{2}}-\frac{p_{0}}{(p-1)^{2}},
$$

we get by the Hölder inequality

$$
\begin{aligned}
E & {\left[\left(\frac{Z_{T}}{Z}\right)^{1 /(p-1)} \mid F_{T}\right] } \\
& =E\left[\exp \left\{-\frac{1}{p-1}\left(M_{\infty}-M_{T}\right)-\frac{p_{0}}{2(p-1)^{2}}\left(\langle M\rangle_{\infty}-\langle M\rangle_{T}\right)\right\}\right.
\end{aligned}
$$




$$
\begin{aligned}
& \left.\times \exp \left\{\frac{1}{2 q_{0}(\sqrt{p}-1)^{2}}\left(\langle M\rangle_{\infty}-\langle M\rangle_{T}\right)\right\} \mid F_{T}\right] \\
= & E\left[\exp \left\{-\frac{p_{0}}{p-1}\left(M_{\infty}-M_{T}\right)-\frac{1}{2}\left(\frac{p_{0}}{p-1}\right)^{2}\left(\langle M\rangle_{\infty}-\langle M\rangle_{T}\right)\right\} \mid F_{T}\right]^{1 / p_{0}} \\
& \times E\left[\exp \left\{\frac{1}{2(\sqrt{p}-1)^{2}}\left(\langle M\rangle_{\infty}-\langle M\rangle_{T}\right)\right\} \mid F_{T}\right]^{1 / q_{0}} .
\end{aligned}
$$

The first term on the right side is smaller than 1 , and by the assumption the second term is dominated by some constant $C_{p}$. Therefore $Z$ satisfies $\left(\mathrm{A}_{\mathrm{p}}\right)$. Similarily, $Z^{\prime}$ satisfies $\left(\mathrm{A}_{p}\right)$. Thus the lemma is established.

Proof of Theorem 1. We begin with the proof of the part (i). Firstly, suppose that $Z$ satisfies $\left(B_{\lambda}\right)$ for all $\lambda>0$. Then it follows from Lemma 3 (iii) that $Z$ and $Z^{\prime}$ satisfy $\left(\mathrm{A}_{\mathrm{p}}\right)$ for all $p>1$. On the other hand, for any $\lambda>0$ we can choose $p>1$ such that $\lambda \leqq 1 /(2(p-1))$. Thus from Lemma 3 (ii) the sufficiency follows at once.

Now we are going to show the part (ii). Assume that $Z$ satisfies $\left(\mathrm{B}_{\lambda}\right)$ for some $\lambda>0$. And choose $p>1$ such that $1 /\left(2(\sqrt{p}-1)^{2}\right) \leqq \lambda$. Then Lemma 3 (iii) implies that $Z$ satisfies $\left(\mathrm{A}_{\mathrm{p}}\right)$. Conversely, if $Z$ satisfies $\left(A_{p}\right)$, then from Lemma 3 (i) it satisfies $\left(B_{\lambda}\right)$ for $\lambda=1 /\left(2(\sqrt{p}+1)^{2}\right)$. The theorem is proved.

As remarked in Section 1 , in order that $Z$ satisfies $\left(B_{\infty}\right)$, it is necessary and sufficient that $M$ is a $B M O$-martingale. Thus we get:

Corollary 1. $Z$ satisfies $\left(\mathrm{A}_{\infty}\right)$ if and only if $M$ is a BMO-martingale.

Corollary 2. Let $p>9$. If $Z$ satisfies $\left(\mathrm{A}_{p_{0}}\right)$ for $p_{0}=(\sqrt{p}-2)^{2}$, then $Z^{\prime}$ satisfies $\left(\mathrm{A}_{\mathrm{p}}\right)$.

Proof. As $\left(\sqrt{p_{0}}+1\right)^{2}=(\sqrt{p}-1)^{2}$, Lemma 3 shows that for any stopping time $T$

$$
E\left[\exp \left\{\frac{1}{2(\sqrt{\bar{p}}-1)^{2}}\left(\langle M\rangle_{\infty}-\langle M\rangle_{T}\right)\right\} \mid F_{T}\right] \leqq C_{p}
$$

Thus $Z^{\prime}$ satisfies $\left(\mathrm{A}_{\mathrm{p}}\right)$.

It is also easy to check that $Z$ satisfies $\left(\mathrm{A}_{\mathrm{p}}\right)$ if $\|M\|_{\text {вмо }}<\sqrt{\overline{2}}(\sqrt{\bar{p}}-1)$.

Proposition. Let $T$ be any stopping time. Suppose that for every $p>1$ there exists a constant $\varepsilon>0$ such that $\varepsilon<p-1$ and

$$
E\left[\exp \left(\frac{2}{p-1-\varepsilon}\left|M_{\infty}-M_{T}\right|\right) \mid F_{T}\right] \leqq C_{p}
$$


Then $Z$ satisfies $\left(\mathrm{A}_{\mathrm{p}}\right)$ for all $p>1$ if and only if $Z^{\prime}$ satisfies $\left(\mathrm{A}_{\mathrm{p}}\right)$ for all $p>1$.

Proof. We only show the necessity. Assume that $Z$ satisfies $\left(\mathrm{A}_{\mathrm{p}}\right)$ for all $p>1$. Set $p_{0}=(p-1) / \varepsilon$. Then the exponent conjugate $q_{0}$ is $(p-1) /(p-1-\varepsilon)$. As $Z_{T}^{\prime} / Z^{\prime}=\exp \left\{2\left(M_{\infty}-M_{T}\right)\right\} Z_{T} / Z$, the Hölder inequality shows that

$$
\begin{aligned}
& E\left[\left(\frac{Z_{T}^{\prime}}{Z^{\prime}}\right)^{1 /(p-1)} \mid F_{T}\right] \\
& \quad \leqq E\left[\left(\frac{Z_{T}}{Z}\right)^{p_{0} /(p-1)} \mid F_{T}\right]^{1 / p_{0}} E\left[\exp \left(\frac{2 q_{0}}{p-1}\left|M_{\infty}-M_{T}\right|\right) \mid F_{T}\right]^{1 / q_{0}} .
\end{aligned}
$$

The first term on the right side is dominated by $A_{r}(Z)^{1 / p_{0}(r-1)}$ where $r=$ $\left(p+p_{0}-1\right) / p_{0}=1+\varepsilon>1$. The second term is also dominated by some constant $C_{p}$, from the assumption. Consequently, $Z^{\prime}$ satisfies $\left(\mathrm{A}_{\mathrm{p}}\right)$.

Particularly, if the martingale $M$ is bounded, the following are equivalent:

$1^{\circ} . \quad Z$ satisfies $\left(\mathrm{A}_{p}\right)$

$2^{\circ}$. $Z^{\prime}$ satisfies $\left(\mathrm{A}_{p}\right)$

$3^{\circ}$. $Z$ satisfies $\left(\mathrm{B}_{\lambda}\right)$ for $\lambda=\frac{1}{2(p-1)}$.

However we do not know whether this equivalence is true in general.

3. A weighted norm inequality for continuous martingales. We use $d \hat{P}$ to denote the weighted probability measure $Z d P$, and let $\hat{E}[\cdot]$ be expectation over $\Omega$ with respect to $d \hat{P}$. As $Z_{t}$ is a $P$-martingale, we get $d \hat{P}=Z_{t} d P$ on each $F_{t}$. Thus an elementary calculation shows that

$$
\hat{E}\left[Y \mid F_{t}\right]=\frac{E\left[Z Y \mid F_{t}\right]}{Z_{t}} \text { a.s., under } d P \text { and } d \hat{P}
$$

for every $\hat{P}$-integrable random variable $Y$.

THEOREM 2. If $Z$ satisfies $\left(\mathrm{A}_{\infty}\right)$, then there exists a non-decreasing sequence $\left(T_{n}\right)$ of stopping times with $\lim _{n} T_{n}=\infty$ such that we have

$$
\hat{E}\left[\left(X_{T_{n}}^{*}\right)^{p}\right] \leqq C_{p, n} \hat{E}\left[\left|X_{T_{n}}\right|^{p}\right], \quad 1<p<\infty, \quad n=1,2, \cdots
$$

for all $P$-uniformly integrable martingale $X$ where $X_{T_{n}}^{*}=\sup _{t}\left|X_{t \wedge T_{n}}\right|$.

Proof. By the assumption, $Z$ satisfies $\left(\mathrm{A}_{p_{0}}\right)$ for some $p_{0}>1$. Let $X$ be any $P$-uniformly integrable martingale, and set $X^{*}=X_{\infty}^{*}$. Firstly, we show that the inequality

$$
\hat{E}\left[\left(X^{*}\right)^{p}\right] \leqq C_{p} \hat{E}\left[\left|X_{\infty}\right|^{p}\right]
$$


is valid for every $p>p_{0}$. To see it, we may assume that $\hat{E}\left[\left|X_{\infty}\right|^{p}\right]$ is finite. As $X_{t}=Z_{t} \hat{E}\left[X_{\infty} / Z \mid F_{t}\right]$, we get

$$
\left|X_{t}\right|^{p_{0}} \leqq\left\{Z_{t}^{p_{0}} \hat{E}\left[(1 / Z)^{q_{0}} \mid F_{t}\right]^{p_{0} / q_{0}}\right\} \hat{E}\left[\left|X_{\infty}\right|^{p_{0}} \mid F_{t}\right]
$$

from the Hölder inequality. The first term on the right side is $E\left[\left(Z_{t} / Z\right)^{1 /\left(p_{0}-1\right)} \mid F_{t}\right]^{p_{0}-1}$ which is dominated by $A_{p_{0}}(Z)$. Then, applying the Doob inequality to the $\hat{P}$-martingale $\left\{\hat{E}\left[\left|X_{\infty}\right|^{p_{0}} \mid F_{t}\right]\right\}_{t \geq 0}$, we have

$$
\begin{aligned}
\hat{E}\left[\left(X^{*}\right)^{p}\right] & \leqq A_{p_{0}}(Z) \hat{E}\left[\sup _{t} \hat{E}\left[\left|X_{\infty}\right|^{p_{0}} \mid F_{t}\right]^{p / p_{0}}\right] \\
& \leqq C_{p} \hat{E}\left[\left|X_{\infty}\right|^{p}\right] .
\end{aligned}
$$

Therefore, if $Z$ satisfies $\left(\mathrm{A}_{\mathrm{p}}\right)$ for all $p>1$, the inequality (10) holds for every $p>1$.

Now let $T_{n}=\inf \left\{t \geqq 0 ;\langle M\rangle_{t}>n\right\}, n=1,2, \cdots$. Then clearly $T_{n} \leqq$ $T_{n+1} \rightarrow \infty$ a.s., $\langle M\rangle_{T_{n}} \leqq n$ and $E\left[Z \mid F_{T_{n}}\right]=Z_{T_{n}}$. Set

$$
Z_{t}^{(n)}=\exp \left(M_{t \wedge T_{n}}-\frac{1}{2}\langle M\rangle_{t \wedge T_{n}}\right) \text {. }
$$

As $\langle M\rangle_{t \wedge T_{n}} \leqq n, Z^{(n)}=Z_{T_{n}}$ satisfies $\left(\mathrm{B}_{\lambda}\right)$ for all $\lambda>0$. Thus it follows from Theorem 1 that each $Z^{(n)}$ satisfies $\left(\mathrm{A}_{p}\right)$ for all $p>1$. Since $\left|X_{T_{n}}\right|$ and $X_{T_{n}}^{*}$ are $F_{T_{n}}$-measurable, we have

$$
\begin{aligned}
\hat{E}\left[\left(X_{T_{n}}^{*}\right)^{p}\right] & =E\left[Z\left(X_{T_{n}}^{*}\right)^{p}\right] \\
& =E\left[Z^{(n)}\left(X_{T_{n}}^{*}\right)^{p}\right] \\
& \leqq C_{p, n} E\left[Z^{(n)}\left|X_{T_{n}}\right|^{p}\right] \\
& =C_{p, n} E\left[Z\left|X_{T_{n}}\right|^{p}\right] \\
& =C_{p, n} \hat{E}\left[\left|X_{T_{n}}\right|^{p}\right]
\end{aligned}
$$

for every $p>1$, completing the proof.

We proved in [1]: if $(1 / Z)^{1 /(p-1)} \in L^{1}(d P)$ and the inequality (10) is valid for all $P$-martingale $X$ with $\sup _{t} \hat{E}\left[\left|X_{t}\right|^{p}\right]<\infty$, then $Z$ satisfies $\left(\mathrm{A}_{\mathrm{p}}\right)$.

Finally, as an application, we give a new martingale inequality. For that, we need the next two lemmas.

LEMMA 4. Let $X$ be any $P$-continuous local martingale, and let $\langle X, M\rangle=(1 / 2)(\langle X+M\rangle-\langle X\rangle-\langle M\rangle)$. Then $\hat{X}=X-\langle X, M\rangle$ is a $\hat{P}$-continuous local martingale such that $\langle\hat{X}\rangle=\langle X\rangle$ under either probability measure.

Proof. We sketch briefly its proof. Because of (8), for $s<t$

$$
\hat{E}\left[\hat{X}_{t} \mid F_{s}\right]=\frac{1}{Z_{s}} E\left[Z_{t} \hat{X}_{t} \mid F_{s}\right] .
$$


Therefore, to see that $\hat{X}=X-\langle X, M\rangle$ is a $\hat{P}$-local martingale, it suffices to show that $Z \hat{X}$ is a $P$-local martingale. Observe that the process $\hat{X}$ is a semi-martingale with respect to $d P$. As $d\langle X, M\rangle_{t}=\left(1 / Z_{t}\right) d\langle X, Z\rangle_{t}$, applying the Ito formula we get

$$
Z_{t} \hat{X}_{t}=Z_{0} \hat{X}_{0}+\int_{0}^{t} \hat{X}_{s} d Z_{s}+\int_{0}^{t} Z_{s} d X_{s}
$$

which is a $P$-local martingale. It is immediate to check that $\langle\hat{X}\rangle=$ $\langle X\rangle$ under $d P$ and $d \hat{P}$.

We now point out that the converse is true. More precisely, for any $\hat{P}$-continuous local martingale $X^{\prime}, X=X^{\prime}+\left\langle X^{\prime}, \hat{M}\right\rangle$ is a $P$-continuous local martingale. From Lemma $4, \hat{X}=X-\langle X, M\rangle$ is a $\hat{P}$-continuous local martingale, so that $\hat{X}-X^{\prime}=\left\langle X^{\prime}, \hat{M}\right\rangle-\langle X, M\rangle$ is also a $\hat{P}$-continuous local martingale zero at $t=0$. Thus $\hat{X}=X^{\prime}$.

Now if $Z^{\prime}$ satisfies the $\left(\mathrm{A}_{2}\right)$ condition, then $\hat{M}=M-\langle M\rangle$ is a $B M O$-martingale with respect to $d \hat{P}$ since $\hat{E}\left[\exp \left(\langle\hat{M}\rangle_{\infty}-\langle\hat{M}\rangle_{T}\right) \mid F_{T}\right]=$ $E\left[Z_{T}^{\prime} / Z^{\prime} \mid F_{T}\right]$ for any stopping time $T$. But we do not know whether $\hat{M}$ is a $B M O$-martingale with respect to $d \hat{P}$ for any $B M O$-martingale $M$.

LEMMA 5. For any $\lambda>0$ and any stopping time $T$,

$$
\begin{aligned}
& \hat{E}\left[\exp \left\{\lambda\left(\langle\hat{M}\rangle_{\infty}-\langle\hat{M}\rangle_{T}\right)\right\} \mid F_{T}\right] \\
& \quad \leqq E\left[\exp \left\{(2 \lambda+1)\left(\langle M\rangle_{\infty}-\langle M\rangle_{T}\right)\right\} \mid F_{T}\right]^{1 / 2} .
\end{aligned}
$$

Proof. Since $\exp \left(2 M_{t}-2\langle M\rangle_{t}\right)$ is a local martingale, an elementary stopping argument gives

$$
E\left[\exp \left\{2\left(M_{\infty}-M_{T}\right)-2\left(\langle M\rangle_{\infty}-\langle M\rangle_{T}\right)\right\} \mid F_{T}\right] \leqq 1 .
$$

As

$$
\begin{aligned}
\left(Z / Z_{T}\right) \exp \left\{\lambda\left(\langle\hat{M}\rangle_{\infty}-\langle\hat{M}\rangle_{T}\right)\right\}=\exp \left\{\left(M_{\infty}-M_{T}\right)-\left(\langle M\rangle_{\infty}-\langle M\rangle_{T}\right)\right\} \\
\times \exp \left\{\frac{2 \lambda+1}{2}\left(\langle M\rangle_{\infty}-\langle M\rangle_{T}\right)\right\},
\end{aligned}
$$

the Schwarz inequality shows (11).

Thus, according to the Jensen inequality, $\hat{M}$ is a $B M O$-martingale with respect to $d \hat{P}$ if $E\left[\exp \left\{\lambda\left(\langle M\rangle_{\infty}-\langle M\rangle_{T}\right)\right\} \mid F_{T}\right] \leqq C_{\lambda}$ for some $\lambda>1$.

THEOREM 3. Suppose that $Z$ satisfies $\left(\mathrm{B}_{\lambda}\right)$ for all $\lambda>0$. Then the inequality

$$
E\left[\sup _{t}\left|X_{t}-\langle X, M\rangle_{t}\right|^{p}\right] \leqq C_{p} E\left[\left|X_{\infty}-\langle X, M\rangle_{\infty}\right|^{p}\right], \quad 1<p<\infty
$$

is valid for all $L^{2}(d P)$-bounded continuous martingale $X$. 
Proof. The process defined by $\hat{Z}_{t}=\exp \left(-\hat{M}_{t}-(1 / 2)\langle\hat{M}\rangle_{t}\right), t \geqq 0$, is a $\hat{P}$-continuous local martingale. But, as $\hat{M}=M-\langle M\rangle$, we get $\hat{Z}_{t}=1 / Z_{t}$ and $\hat{Z}=\hat{Z}_{\infty}=1 / Z \in L^{1}(d \hat{P})$, so that $\hat{E}\left[\hat{Z} \mid F_{t}\right]=\hat{Z}_{t}$ from (8). That is to say, $\hat{Z}_{t}$ is $\hat{P}$-uniformly integrable. As $\hat{Z} d \hat{P}=d P$, we also get from Lemma 5

$$
E\left[\exp \left\{\lambda\left(\langle M\rangle_{\infty}-\langle M\rangle_{T}\right)\right\} \mid F_{T}\right] \leqq \hat{E}\left[\exp \left\{(2 \lambda+1)\left(\langle\hat{M}\rangle_{\infty}-\langle\hat{M}\rangle_{T}\right)\right\} \mid F_{T}\right]^{1 / 2} .
$$

Therefore $Z$ satisfies $\left(B_{\lambda}\right)$ for all $\lambda>0$ if and only if $\hat{Z}$ satisfies $\left(B_{\lambda}\right)$ for all $\lambda>0$ with respect to $d \hat{P}$. Then, from the proof of Theorem 2 , if $Z$ satisfies $\left(B_{2}\right)$ for all $\lambda>0$, the inequality

$$
E\left[\left(X^{* *}\right)^{p}\right] \leqq C_{p} E\left[\left|X_{\infty}^{\prime}\right|^{p}\right], \quad 1<p<\infty
$$

is valid for all $\hat{P}$-uniformly integrable martingale $X^{\prime}$. Thus, to prove (12), it suffices to show that $\hat{X}=X-\langle X, M\rangle$ is a $\hat{P}$-uniformly integrable martingale for every $L^{2}(d P)$-bounded continuous martingale $X$. By the Davis inequality and Lemma 4, we have

$$
\frac{1}{4 \sqrt{2}} \hat{E}\left[\hat{X}^{*}\right] \leqq \hat{E}\left[\langle X\rangle_{\infty}^{1 / 2}\right] \text {. }
$$

By the Schwarz inequality the right side is smaller than $E\left[Z^{2}\right]^{1 / 2} E\left[X_{\infty}^{2}\right]^{1 / 2}$. As $Z$ satisfies $\left(B_{\lambda}\right)$ for all $\lambda>0$, Lemma 1 shows that

$$
E\left[Z^{2}\right] \leqq E\left[\exp \left(2\left|M_{\infty}\right|\right)\right]<\infty .
$$

Thus for any $L^{2}(d P)$-bounded continuous martingale $X, \hat{X}^{*}$ is $\hat{P}$-integrable. Consequently, $\hat{X}$ is $\hat{P}$-uniformly integrable. This completes the proof.

More generally, let $X$ be a $P$-continuous local martingale. Since there exist stopping times $T_{n} \uparrow \infty$ a.s., such that for each $n$ the process $\left(X_{t \wedge T_{n}}\right)$ stopped at $T_{n}$ is an $L^{2}(d P)$-bounded martingale, we obtain

$$
\begin{aligned}
E\left[\sup _{t}\left|X_{t}-\langle X, M\rangle_{t}\right|^{p}\right] \leqq C_{p} \sup _{n} E\left[\left|X_{T_{n}}-\langle X, M\rangle_{T_{n}}\right|^{p}\right], & 1<p<\infty
\end{aligned}
$$

from the Fatou lemma.

We can weaken the above assumption on $Z$ as follows.

THEOREM 4. Suppose that $Z$ satisfies $\left(\mathrm{A}_{\infty}\right)$. Let $1<p<\infty$. Then there exists a constant $c_{p}>0$ depending only on $p$ such that the inequality

$$
E\left[\sup _{t}\left|X_{t}-c_{p}\langle X, M\rangle_{t}\right|^{p}\right] \leqq C_{p} E\left[\left|X_{\infty}-c_{p}\langle X, M\rangle_{\infty}\right|^{p}\right]
$$

is valid for all $L^{2}(d P)$-bounded continuous martingale $X$.

Proof. If $Z$ satisfies $\left(\mathrm{A}_{\infty}\right)$, then it follows from Theorem 1 that $Z$ 
satisfies $\left(B_{\lambda_{0}}\right)$ for some $\lambda_{0}>0$. Then choose $\lambda>0$ such that

$$
\lambda>\operatorname{Max}\left\{1+\frac{1}{(\sqrt{p}-1)^{2}}, 8 \lambda_{0}\|M\|_{B . U O}^{2}\right\}
$$

and set $N=c_{p} M, c_{p}=\left(\lambda_{0} / \lambda\right)^{1 / 2}$. The process $N$ is a $B M O$-martingale. As $\langle N\rangle=c_{p}^{2}\langle M\rangle$, we get

$$
\|\sqrt{8} N\|_{B M O}^{2}=8 c_{p}^{2}\|M\|_{B M O}^{2}=\frac{1}{\lambda} 8 \lambda_{0}\|M\|_{B M O}^{2}<1 .
$$

Now we define $Z_{t}^{*}=\exp \left(N_{t}-(1 / 2)\langle N\rangle_{t}\right)$ and $Z^{\#}=Z_{\infty}^{\#} . \quad A s\|\sqrt{8} N\|_{B M O}^{2}<1$, Lemma 2 implies that $Z^{\#}$ satisfies the $\left(B_{8}\right)$ condition. Thus by Lemma 1 the process $Z_{t}^{\#}$ is an $L^{2}(d P)$-bounded martingale, so that $d \hat{P}=Z^{\sharp} d P$ is a probability measure on $\Omega$. Set $\alpha=(\lambda-1) / 2>0$. Then from Lemma 5 we get

$$
\begin{aligned}
\hat{E}\left[\exp \left\{\alpha\left(\langle\hat{N}\rangle_{\infty}-\langle\hat{N}\rangle_{T}\right)\right\} \mid F_{T}\right] & \leqq E\left[\exp \left\{\lambda\left(\langle N\rangle_{\infty}-\langle N\rangle_{T}\right)\right\} \mid F_{T}\right]^{1 / 2} \\
& \leqq E\left[\exp \left\{\lambda_{0}\left(\langle M\rangle_{\infty}-\langle M\rangle_{T}\right)\right\} \mid F_{T}\right]^{1 / 2}
\end{aligned}
$$

where $\hat{N}=N-\langle N\rangle$. The right side is dominated by some constant $C$ because $Z$ satisfies $\left(\mathrm{B}_{\lambda_{0}}\right)$. As $\alpha>1 / 2(\sqrt{p}-1)^{2}$, we have

$$
\hat{E}\left[\exp \left\{\frac{1}{2(\sqrt{p}-1)^{2}}\left(\langle\hat{N}\rangle_{\infty}-\langle\hat{N}\rangle_{T}\right)\right\} \mid F_{T}\right] \leqq C .
$$

Thus from Lemma $3, \hat{Z}^{\#}$ satisfies $\left(\mathrm{A}_{\mathrm{p}}\right)$ with respect to $d \hat{P}$ where $\hat{Z}_{t}^{\#}=$ $\exp \left(-\hat{N}_{t}-(1 / 2)\langle\hat{N}\rangle_{t}\right)$ and $\hat{Z}^{\sharp}=\hat{Z}_{\infty}^{\sharp}$ as before. The process $\hat{Z}_{t}^{\sharp}$ is a $\hat{P}$ uniformly integrable continuous martingale such that $\hat{Z}_{t}^{\sharp}=1 / Z_{t}^{\sharp}$. Furthermore, one can choose $\varepsilon>0$ such that $\varepsilon<p-1$ and $\alpha>1 / 2(\sqrt{p-\varepsilon}-1)^{2}$. Then $\hat{Z}^{\#}$ satisfies the $\left(\mathrm{A}_{\mathrm{p}-\varepsilon}\right)$ condition. Therefore the inequality

$$
E\left[\left(X^{*}\right)^{p}\right] \leqq C_{p} E\left[\left|X_{\infty}^{\prime}\right|^{p}\right]
$$

is valid for all $\hat{P}$-uniformly integrable martingale $X^{\prime}$. And, as the martingale $Z_{t}^{\sharp}$ is $L^{2}(d P)$-bounded, $\hat{X}=X-\langle X, N\rangle=X-c_{p}\langle X, M\rangle$ is a $\hat{P}$-uniformly integrable martingale for any $L^{2}(d P)$-bounded continuous martingale $X$. This completes the proof.

\section{REFERENCES}

[1] M. Izumisama AND N. Kazamaki, Weighted norm inequalities for martingales, Tôhoku Math. J., 29 (1977), 115-124.

[2] P. A. Meyer, Intégrales stochastiques I, II, Séminaire de Probabilités I, Lecture Notes in Math. 39. Springer-Verlag (1967).

College of General Education

TÔHOKU UNIVERSITY

SENDAI, JAPAN 
